\title{
Diseño e implementación de un Plan de Requerimiento de Materiales, Kárdex y un Plan de Mantenimiento Preventivo en una empresa embotelladora de agua de mesa para reducir sus costos operacionales
}

\author{
Design and Implementation of a Materials Requirement Plan, a \\ Preventive Maintenance Plan and Kardex in a Table Water Bottling \\ Company to Reduce its Operational Cost
}

\author{
Rafael Castillo, Maestro en Dirección de Operaciones y Cadena de Abastecimiento ${ }^{1}$, Alejandra Llanos, Ingeniera \\ Industrial $^{2}$ \\ ${ }^{1}$ Universidad Privada del Norte, Perú, rafael.castillo@upn.edu.pe \\ ${ }^{2}$ Universidad Privada del Norte, Perú, malejandra.1lanosc@gmail.com
}

\begin{abstract}
Resumen-El presente trabajo fue elaborado con el objetivo de implementar distintas herramientas de ingeniería en la empresa embotelladora de agua EBERIA S.A.C. de la ciudad de Trujillo, Perú. El trabajo tuvo una etapa inicial de diagnóstico, donde se identificaron distintos problemas como demanda insatisfecha, paradas de máquina, devolución y extravio de bidones. Para la solución de estos, se propusieron dos alternativas de solución, las cuales fueron evaluadas con restricciones realistas y se seleccionó la más adecuada, con el fin de determinar que herramientas se adecuaban mejor al contexto actual de la empresa. Las herramientas implementadas fueron Plan de Requerimiento de Materiales (MRP), Mantenimiento preventivo y Kárdex y se compararon los resultados con estándares apropiados de ingeniería para determinar su impacto. Finalmente se obtuvo una reducción anual de S/ 5,231.41 en costos y a la vez se evaluó el impacto de la implementación de las herramientas elaboradas, obteniendo un TIR de 29\%, un VAN de S/3,496.00 y un B/C de 3.05.
\end{abstract}

Palabras clave - MRP, Kárdex, Mantenimiento Preventivo, Embotelladora, Implementación.

Abstract - The present work was prepared with the objective of implementing different engineering tools in the water bottling company EBERIA S.A.C. from the city of Trujillo, Peru. The work had an initial stage of diagnosis, where different problems were identified as unsatisfied demand, machine stops, return and loss of water barrel. For the solution of these, two solution alternatives were proposed, which were evaluated with realistic constraints and the most appropriate were selected, in order to determine which tools best suited the current context of the company. The implemented tools were Materials Requirement Plan, Preventive Maintenance and Kardex and the results were compared with appropriate engineering standards to determine their impact. Finally, it was obtained an annual reduction of $S / 5,231.41$ in costs and at the same time the impact of the implementation of the tools developed was evaluated, obtaining an IRR of 29\%, NPV of S /3,496.00 and $a \mathrm{~B} / \mathrm{C}$ of 3.05.

Key Words - MRP, Kardex, Preventive Maintenance, Bottler, Implementation

\begin{tabular}{l} 
Digital Object Identifier (DOI): \\
http://dx.doi.org/10.18687/LACCEI2021.1.1.85 \\
ISBN: $978-958-52071-8-9$ ISSN: 2414-6390 \\
\hline
\end{tabular}

\section{INTRODUCCIÓN}

\section{A. Realidad Problemática}

La empresa donde se realizó la presente investigación se dedica al tratamiento, embotellamiento y comercialización de agua de mesa en el norte de Perú desde el año 2015. Durante los meses de Enero, Febrero y Marzo del año 2019, se produjeron y comercializaron 415 botellones de 7 litros, 30 bidones descartables de 21 litros y 18,339 bidones retornables de 20 litros, siendo este último su producto más vendido.

Durante el transcurso de estos 04 años, la empresa ha podido posicionarse en el mercado del norte del país, implementando áreas administrativas que han podido dar soporte a las operaciones, lo cual les ha permitido cumplir los estándares de calidad que exige el mercado. A pesar del crecimiento mencionado y calidad de sus productos, la empresa ha tenido distintos problemas que han afectado directamente a su rentabilidad, por los sobrecostos ocasionados por algunos problemas; entre ellos el incumplimiento de demanda, paradas de maquinaria y extravío de sus bidones retornables de 20 litros, como se muestra en la Tabla 1.

El incumplimiento de la demanda es ocasionado por los sobretiempos en la producción por parte de los operarios, quienes tienen distintas capacidades de acuerdo con su experiencia y habilidades, por lo que la empresa no puede establecer de manera clara los pronósticos diarios de producción ni horas de trabajo a asignar, esto por la falta de estandarización de procesos. El costo generado por este problema fue de S/ 4,012.66 durante el año 2018.

Por otro lado, existen paradas de maquinaria porque la empresa solamente aplica mantenimiento correctivo cuando alguna máquina presenta fallas; estas reparaciones usualmente toman entre 90 y 120 minutos. Estas paradas afectan la vida útil de la maquinaria y ocasionan tiempo muerto de los operarios, quienes dejan de trabajar durante las reparaciones. El costo generado por este problema fue de S/ 509.50 durante el año 2018.

$19^{\text {th }}$ LACCEI International Multi-Conference for Engineering, Education, and Technology: "Prospective and trends in technology and skills for sustainable social development" "Leveraging emerging technologies to construct the future", Buenos Aires -Argentina, July 21-23, 2021. 
Finalmente, el extravío de bidones retornables de 20 litros se da porque la empresa no cuenta con un registro escrito de los bidones que son prestados a los distribuidores y/o consumidores finales, ni con una persona que haga el seguimiento respectivo. El costo generado por este problema fue de S/ 4,163.72 durante el año 2018.

TABLA 1

RESUMEN DE COSTOS DE PROBLEMAS

\begin{tabular}{|c|l|}
\hline Problema & Costo 2018 \\
\hline Incumplimiento de demanda & S/ 4,012.66 \\
\hline Paradas de maquinaria & S/ 509.50 \\
\hline Extravío de bidones & S/ 4,163.72 \\
\hline
\end{tabular}

Cáceres, en su tesis "Propuesta de mejora de la eficiencia global de los equipos orientado en el TPM para una empresa envasadora de bebida gasificada no alcohólica" obtuvo un aumento del OEE de $67.6 \%$ hasta $76.2 \%$, utilizando el pilar de Mantenimiento Autónomo perteneciente al TPM, el cual utiliza un Plan de Mantenimiento Preventivo [11].

Llontop, Viacava y Málaga, en su tesis "Propuesta de mejora del proceso de producción en una planta embotelladora de productos de consumo masivo mediante técnicas Lean" obtuvieron una mejora en la eficiencia de materiales de $2.25 \%$, lo cual contribuyó al cumplimiento de la demanda [12].

Moraida, en su tesis "Implementación de procesos de control de inventarios y su impacto en la rentabilidad de la empresa metal mecánica SERMETAL S.A.C., del distrito de Pataz - La Libertad, 2016", obtuvo una reducción de 14\% en los extravíos de materiales mediante el uso de procesos de control de inventarios, incluyendo Kardex [13].

\section{B. Problema}

¿En qué medida el diseño e implementación de un MRP, Kárdex y un Plan de Mantenimiento Preventivo en una empresa embotelladora de agua de mesa reduce sus costos operacionales?

\section{Objetivo}

Determinar en qué medida se pueden reducir los costos operacionales de una empresa embotelladora de agua de mesa a través del diseño e implementación de un MRP, Kárdex y un Plan de Mantenimiento Preventivo.

\section{MATERIALES Y MÉTODOS}

\section{A. Diseño de la Investigación}

La presente investigación es de carácter Experimental de grado Pre - Experimental, donde la unidad de estudio fue el proceso de embotellamiento de agua de la empresa Corporación Eberia S.A.C. y la población fue constituida por 3 procesos y 4 trabajadores en planta de dicha empresa.

\section{B. Alternativas de Solución}

Para poder resolver los problemas mencionados anteriormente, se plantearon dos alternativas integrales de solución:

TABLA 2

RESUMEN DE ALTERNATIVAS DE SOLUCIÓN

\begin{tabular}{|c|c|c|}
\hline Problema & \multicolumn{2}{|c|}{ Alternativas de solución } \\
\hline $\begin{array}{c}\text { Incumplimiento } \\
\text { de la demanda }\end{array}$ & $\begin{array}{c}\text { Implementación de un } \\
\text { Plan de Requerimiento } \\
\text { de Materiales }\end{array}$ & $\begin{array}{c}\text { Implementación de un } \\
\text { Balanceo de Línea }\end{array}$ \\
\hline $\begin{array}{c}\text { Paradas de } \\
\text { Maquinaria }\end{array}$ & $\begin{array}{c}\text { Implementación de un } \\
\text { Plan de } \\
\text { Mantenimiento }\end{array}$ & $\begin{array}{c}\text { Implementación de } \\
\text { Mantenimiento } \\
\text { Preventivo Total }\end{array}$ \\
\hline $\begin{array}{c}\text { Extravío de } \\
\text { Bidones }\end{array}$ & $\begin{array}{c}\text { Implementación de } \\
\text { Kárdex }\end{array}$ & $\begin{array}{c}\text { Implementación de } \\
\text { Gestión de Inventarios }\end{array}$ \\
\hline
\end{tabular}

\section{Identificación y descripción de Restricciones Realistas}

Para escoger la alternativa de solución más idónea para cada uno de los problemas, se evaluaron a través de restricciones realistas, las cuales funcionan como limitaciones que sirven de guía al momento de seleccionar la mejor, tomando en cuenta el objetivo del trabajo.

TABLA 3

CUADRO COMPARATIVO DE ALTERNATIVAS DE SOLUCIÓN DE INCUMPLIMIENTO DE LA DEMANDA

\begin{tabular}{|c|c|c|}
\hline Restricciones & Implementación de MRP & $\begin{array}{c}\text { Implementación de } \\
\text { Balance de Línea }\end{array}$ \\
\hline $\begin{array}{c}\text { Costo de } \\
\text { Implementación }\end{array}$ & S/ 750.00 & S/ 3342.00 \\
\hline $\begin{array}{c}\text { Tiempo de } \\
\text { Implementación }\end{array}$ & 10 semanas & 11 semanas \\
\hline Accesibilidad & $\begin{array}{c}\text { Sí es accesible según el } \\
\text { criterio del Gerente } \\
\text { General. }\end{array}$ & $\begin{array}{c}\text { No es accesible según el } \\
\text { criterio del Gerente } \\
\text { General. }\end{array}$ \\
\hline Eficiencia & $100 \%$ & $2 \%$ \\
\hline Sostenibilidad & $\begin{array}{c}\text { Sí es sostenible según } \\
\text { Gerente General }\end{array}$ & $\begin{array}{c}\text { Sí es sostenible según } \\
\text { Gerente General }\end{array}$ \\
\hline Aceptación & $\begin{array}{c}81 \% \text { según trabajadores } \\
\text { de la planta }\end{array}$ & $\begin{array}{c}94 \% \text { según trabajadores de } \\
\text { la planta }\end{array}$ \\
\hline
\end{tabular}

TABLA 4

CUADRO COMPARATIVO DE ALTERNATIVAS DE SOLUCIÓN DE PARADAS DE MAQUINARIA

\begin{tabular}{|c|c|c|}
\hline Restricciones & $\begin{array}{c}\text { Mantenimiento } \\
\text { Preventivo }\end{array}$ & Metodología TPM \\
\hline $\begin{array}{c}\text { Costo de } \\
\text { Implementación }\end{array}$ & S/ 590.00 & S/ 4,400.00 \\
\hline $\begin{array}{c}\text { Tiempo de } \\
\text { Implementación }\end{array}$ & 4 semanas & 10 semanas \\
\hline Ergonomía & $\begin{array}{c}\text { Categoría de Riesgo } \\
\text { Carga: } 4 \\
\text { Descarga: } 4\end{array}$ & $\begin{array}{c}\text { Categoría de Riesgo } \\
\text { Carga: } 4 \\
\text { Descarga: } 4\end{array}$ \\
\hline Confiabilidad & $93.56 \%$ & $94 \%$ \\
\hline Accesibilidad & $\begin{array}{c}\text { Sí es sostenible según } \\
\text { criterios del Gerente } \\
\text { General }\end{array}$ & $\begin{array}{c}\text { Sí es sostenible según } \\
\text { criterios del Gerente } \\
\text { General }\end{array}$ \\
\hline Aceptación & $\begin{array}{c}\text { Sí es accesible según el } \\
\text { criterio del Gerente } \\
\text { General. }\end{array}$ & $\begin{array}{c}\text { Sí es accesible según el } \\
\text { criterio económico del } \\
\text { Gerente General. }\end{array}$ \\
\hline
\end{tabular}


TABLA 5

CUADRO COMPARATIVO DE ALTERNATIVAS DE SOLUCIÓN DE EXTRAVÍO DE BIDONES

\begin{tabular}{|c|c|c|}
\hline Restricciones & $\begin{array}{c}\text { Implementación de } \\
\text { Kárdex }\end{array}$ & $\begin{array}{c}\text { Implementación de } \\
\text { Gestión de Inventarios }\end{array}$ \\
\hline $\begin{array}{c}\text { Costo de } \\
\text { Implementación }\end{array}$ & S/ 131.75 & S/ 224.75 \\
\hline $\begin{array}{c}\text { Tiempo de } \\
\text { Implementación }\end{array}$ & 3 semanas & 3 semanas \\
\hline Accesibilidad & $\begin{array}{c}\text { Sí es accesible según el } \\
\text { criterio del Gerente } \\
\text { General. }\end{array}$ & $\begin{array}{c}\text { Sí es accesible según el } \\
\text { criterio económico del } \\
\text { Gerente General. }\end{array}$ \\
\hline Efectividad & $100 \%$ & $100 \%$ \\
\hline $\begin{array}{c}\text { Costo de } \\
\text { Sostenibilidad }\end{array}$ & S/ 126.00 & S/ 126.00 \\
\hline Aceptación & $\begin{array}{c}100 \% \text { según } \\
\text { trabajadores de la planta }\end{array}$ & $\begin{array}{c}67 \% \text { según trabajadores } \\
\text { de la planta }\end{array}$ \\
\hline
\end{tabular}

\section{Selección de la mejor alternativa}

Después de realizar un análisis comparativo de ambas propuestas de mejora para cada problema encontrado, se escogió:

Para el primer problema de Incumplimiento de la demanda se escogió la elaboración e implementación de un Plan de Requerimiento de Materiales, el cual es un método lógico y fácil de entender para abordar el problema de determinar el número de piezas, componentes y materiales necesarios para producir cada pieza final. MRP también proporciona un programa para especificar cuándo hay que producir o pedir estos materiales, piezas y componentes [1].

Para el problema de las Paradas de Maquinaria se escogió la elaboración e implementación de un Plan de Mantenimiento Preventivo, el cual es el mantenimiento que tiene por misión mantener un nivel de servicio determinado en los equipos, programando las correcciones de sus puntos vulnerables en el momento más oportuno [2].

Para el tercer problema de Extravío de Bidones se escogió como solución la elaboración e implementación de un Kárdex, en donde se lleva el registro de cada unidad, su valor de compra, la fecha de adquisición, el valor de la salida de cada unidad y la fecha en que se retira del inventario. De esta forma, en todo momento se puede conocer el saldo exacto de los inventarios y el valor del costo de venta, el control permanente de los sistemas en base a los inventarios existentes [3].

Para escoger la alternativa de solución más idónea para cada uno de los problemas, se evaluaron a través de restricciones realistas, las cuales funcionan como limitaciones que sirven de guía al momento de seleccionar la mejor, tomando en cuenta el objetivo del trabajo.

\section{DISEÑO}

\section{A. Plan de Requerimiento de Materiales}

El MRP fue diseñado con el objetivo de controlar y coordinar los materiales necesarios, así como para no contar con un inventario excesivo [8].

\section{1) Paso 1: Pronóstico Estacional.}

Implementar un mantenimiento preventivo con el fin de mantener la parte mecánica de la máquina Filtro Multimedia para su operatividad cuando la parte electrónica de la máquina esté en óptimas condiciones.

TABLA 6

PRONÓSTICO SUAVIZADO EXPONENCIAL

\begin{tabular}{|c|c|c|c|c|}
\hline Mes & Mes & Ventas & Pronóstico & DAM \\
\hline 1 & Noviembre & 5,114 & 4,849 & 265 \\
\hline 2 & Diciembre & 4,930 & 5,088 & 158 \\
\hline 3 & Enero & 6,113 & 4,946 & 1167 \\
\hline 4 & Febrero & 6,256 & 5,996 & 260 \\
\hline 5 & Marzo & 6,370 & 6,230 & 140 \\
\hline 6 & Abril & 5,614 & 6,356 & 742 \\
\hline 7 & Mayo & 4,230 & 5,688 & 1458 \\
\hline 8 & Junio & 3,580 & 4,376 & 796 \\
\hline 9 & Julio & 3,685 & 3,660 & 25 \\
\hline 10 & Agosto & 3,158 & 3,682 & 524 \\
\hline 11 & Setiembre & 4,213 & 3,210 & 1003 \\
\hline 12 & Octubre & 4,926 & 4,113 & 813 \\
\hline & & & 4,845 & 612.59 \\
\hline
\end{tabular}

TABLA 7

DEMANDA PRONOSTICADA EN LITROS

\begin{tabular}{|c|c|c|c|c|c|c}
\hline Año & Jul & Ago & Set & Oct & Nov & Dic \\
\hline 2017 & 2,725 & 2,258 & 3,253 & 3,446 & 4,134 & 4,810 \\
\hline 2018 & 3,165 & 2,738 & 3,973 & 4,006 & 4,514 & 4,930 \\
\hline 2019 & 3,685 & 3,158 & 4,213 & 4,926 & 4,845 & 5,230 \\
\hline
\end{tabular}

Se utiliza el método de pronóstico estacional para demostrar que la demanda de la data histórica también es dependiente del periodo. Luego de realizar una regresión lineal para verificar la relación entre ambas variables, se obtuvo un coeficiente de determinación $\mathrm{r}^{\wedge} 2 \quad 0.73$, valor cercano a 1 y que debe ser mayor o igual a 0.7 [4]; por ello, se puede utilizar la data de cualquier año, ya que tienen el mismo comportamiento. 


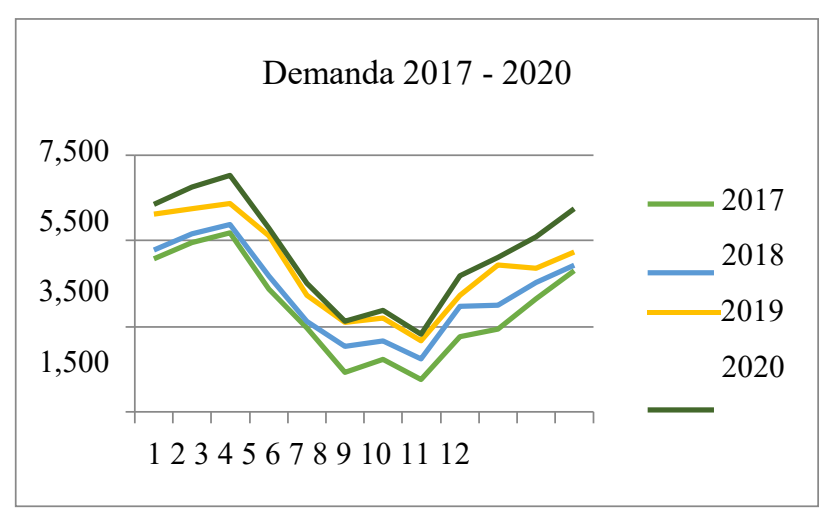

Fig. 1. Demanda del año 2017 al 2019

2) Paso 2: Pronóstico Agregado.

Se elaboró el pronóstico agregado con los datos del pronóstico estacional para determinar el total en litros del siguiente mes de producción.

TABLA 8

DEMANDA AGREGADA EN LITROS

\begin{tabular}{|c|c|c|c|c|c|c|}
\hline LITROS & Jul & Agos & Sept & Oct & Nov & Dic \\
\hline Inventario inicial & 600 & 183 & 184 & 161 & 206 & 242 \\
\hline Demanda & 3,660 & 3,682 & 3,210 & 4,113 & 4,845 & 4,930 \\
\hline Stock de seguridad & 183 & 184 & 161 & 206 & 242 & 247 \\
\hline Req de producción & 3,243 & 3,684 & 3,187 & 4,158 & 4,881 & 4,934 \\
\hline Inventario final & 183 & 184 & 161 & 206 & 242 & 247 \\
\hline
\end{tabular}

3) Paso 3: Plan Maestro de Producción.

Se elaboró el Plan Maestro de Producción con las unidades tomadas del plan agregado, con la finalidad de definir las cantidades y momentos de fabricación mostrado en semanas.

Se tomó en cuenta el pronóstico del mes de noviembre, el cual está expresado en unidades de bidones. Además, se considera que 5 bidones de $20 \mathrm{~L}$ constituyen un lote, es decir, por lote se producen y envasan 100 litros. La empresa para el mes de noviembre consideró un stock de seguridad de 6 bidones y la capacidad de producción de la planta es de 4000 litros por día.

\section{4) Paso 4: Registro de Inventario}

Se define las cantidades de los componentes y materiales que existen en la empresa actualmente. También se muestra el stock de seguridad, lead time, tamaño de lote y entradas previstas de cada uno de ellos.
5) Paso 5: Bill of Materials

TABLA 9

LISTA DE MATERIALES

\begin{tabular}{|c|c|c|}
\hline BIDÓN B20 & CANTIDAD BASE & HECTOLITROS \\
\hline Agua Tratada & Batch & 4.99995 \\
\hline Bidón & Unidad & 5 \\
\hline Caño & Unidad & 5 \\
\hline Etiqueta & Unidad & 5 \\
\hline Precinto & Unidad & 5 \\
\hline Agua Tratada & CANTIDAD BASE & 1 BATCH \\
\hline Agua & L & 20 \\
\hline Cloro & L & 0.0002 \\
\hline & Total & 20.0002 \\
\hline
\end{tabular}

\section{6) Paso 6: Plan de Requerimiento de Materiales}

Se realiza el Plan de Requerimiento de Materiales con la finalidad de saber la cantidad necesaria (cantidad neta) a pedir de manera semanal. Se tiene en cuenta el tamaño de lote y el lead time al momento de programar cada una de las necesidades para la semana.

TABLA 10

MRP - COMPONENTE AGUA TRATADA

\begin{tabular}{|c|c|c|c|c|c|}
\hline \multicolumn{7}{|c|}{ AGUA TRATADA (BATCH) } \\
\hline ¿Quién lo requiere? & Batch/Hectolitro & 1 & 2 & 3 & 4 \\
\hline Bidón 20L & 0.20 & 48.80 & 49.40 & 48.80 & 48.80 \\
\hline Período & Inicial & 1 & 2 & 3 & 4 \\
\hline Necesidades Brutas & & 48.80 & 49.40 & 48.80 & 48.80 \\
\hline Entradas Previstas & & 0.00 & 0.00 & 0.00 & 0.00 \\
\hline Inventario final & 0.00 & 0.00 & 0.00 & 0.00 & 0.00 \\
\hline Necesidades Netas & & 48.80 & 49.40 & 48.80 & 48.80 \\
\hline Pedidos Planeados & & 48.80 & 49.40 & 48.80 & 48.80 \\
\hline $\begin{array}{c}\text { Lanzamiento de } \\
\text { ordenes }\end{array}$ & & 48.80 & 49.40 & 48.80 & 48.80 \\
\hline
\end{tabular}

TABLA 11

MRP - MATERIAL BIDÓN

\begin{tabular}{|c|c|c|c|c|c|}
\hline \multicolumn{5}{|c|}{ BIDÓN } \\
\hline ¿Quién lo requiere? & Unid/Hectolitro & 1 & 2 & 3 & 4 \\
\hline Bidón 20L & 5.00 & 1,220 & 1,235 & 1,220 & 1,220 \\
\hline Período & Inicial & 1 & 2 & 3 & 4 \\
\hline Necesidades Brutas & & 1,220 & 1,235 & 1,220 & 1,220 \\
\hline Entradas Previstas & & 200 & 0.00 & 0.00 & 0.00 \\
\hline Inventario final & 750 & 0.00 & 165 & 145 & 125 \\
\hline Necesidades Netas & & 0.00 & 1,235 & 1,055 & 1,075 \\
\hline Pedidos Planeados & & 0.00 & 1,400 & 1,200 & 1,200 \\
\hline $\begin{array}{c}\text { Lanzamiento de } \\
\text { ordenes }\end{array}$ & & 1,400 & 1,200 & 1,200 & 0 \\
\hline
\end{tabular}


TABLA 12

MRP - MATERIAL CAÑO

\begin{tabular}{|c|c|c|c|c|c|}
\hline \multicolumn{5}{|c|}{ CAÑO } \\
\hline ¿Quién lo requiere? & Unid/Hectolitro & 1 & 2 & 3 & 4 \\
\hline Bidón 20L & 5.00 & 1,220 & 1,235 & 1,220 & 1,220 \\
\hline Período & Inicial & 1 & 2 & 3 & 4 \\
\hline Necesidades Brutas & & 1,220 & 1,235 & 1,220 & 1,220 \\
\hline Entradas Previstas & & 450 & 0.00 & 0.00 & 0.00 \\
\hline Inventario final & 2,000 & 1,230 & 445 & 125 & 255 \\
\hline Necesidades Netas & & 0.00 & 5 & 775 & 1,095 \\
\hline Pedidos Planeados & & 0.00 & 450 & 900 & 1,350 \\
\hline $\begin{array}{c}\text { Lanzamiento de } \\
\text { ordenes }\end{array}$ & & 450 & 900 & 1,350 & 0 \\
\hline
\end{tabular}

TABLA 13

MRP - MATERIAL ETIQUETA

\begin{tabular}{|c|c|c|c|c|c|}
\hline \multicolumn{7}{|c|}{ ETIQUETA } \\
\hline ¿Quién lo requiere? & Unid/Hectolitro & 1 & 2 & 3 & 4 \\
\hline Bidón 20L & 5.00 & 1,220 & 1,235 & 1,220 & 1,220 \\
\hline Período & Inicial & 1 & 2 & 3 & 4 \\
\hline Necesidades Brutas & & 1,220 & 1,235 & 1,220 & 1,220 \\
\hline Entradas Previstas & 200 & 500 & 0.00 & 0.00 & 0.00 \\
\hline Inventario final & & 480 & 245 & 25 & 305 \\
\hline Necesidades Netas & & 520 & 755 & 975 & 1,195 \\
\hline Pedidos Planeados & & 1,000 & 1,000 & 1,000 & 1,500 \\
\hline $\begin{array}{c}\text { Lanzamiento de } \\
\text { ordenes }\end{array}$ & & 1,000 & 1,000 & 1,500 & 0 \\
\hline
\end{tabular}

Para los materiales de precinto, agua y cloro no se lanzarán órdenes en el mes de noviembre por el tamaño de lote o Lead Time.

\section{7) Paso 7: Órdenes de Aprovisionamiento}

Se resume en una lista todas las cantidades necesarias de los materiales cada semana, con los datos obtenidos del MRP.

TABLA 14

PROGRAMA DE COMPRAS PARA EL APROVISIONAMIENTO DE MATERIALES

\begin{tabular}{|c|c|c|c|c|}
\hline \multirow{2}{*}{$\begin{array}{c}\text { PROGRAMA DE } \\
\text { PRODUCCIÓN }\end{array}$} & \multicolumn{4}{|c|}{ SEMANA } \\
\cline { 2 - 5 } & 1 & 2 & 3 & 4 \\
\hline Agua Tratada & 48.80 & 49.40 & 48.80 & 48.80 \\
\hline $\begin{array}{c}\text { PROGRAMA DE } \\
\text { COMPRAS }\end{array}$ & \multicolumn{5}{|c|}{ SEMANA } \\
\cline { 2 - 5 } & 1 & 2 & 3 & 4 \\
\hline Bidón & 1,400 & 1,200 & 1,200 & 0 \\
\hline Caño & 450 & 900 & 1,350 & 0 \\
\hline Etiqueta & 1,000 & 1,000 & 1,500 & 0 \\
\hline Precinto & 0 & 0 & 0 & 0 \\
\hline Agua & 0 & 0 & 0 & 0 \\
\hline Cloro & 0 & 0 & 0 & 0 \\
\hline
\end{tabular}

Para el problema de Incumplimiento de la demanda se utilizó el estándar según el último "Informe de Mercado de Agua Embotellada", la producción de agua embotellado va a crecer $7.9 \%$ este año [7]. Con ello, deducimos que la producción debe de aumentar en un mismo porcentaje para poder satisfacer la demanda.

TABLA 15

ESTÁNDAR DE INCUMPLIMIENTO DE LA DEMANDA

\begin{tabular}{|c|c|c|c|c|c|}
\hline Problema & Estándar & Indicador & Fórmula & V.A & V.E \\
\hline $\begin{array}{c}\text { Incumplim } \\
\text { iento de } \\
\text { demanda } \\
\text { por } \\
\text { desabasteci } \\
\text { miento de } \\
\text { insumos }\end{array}$ & $\begin{array}{c}\text { Informe de } \\
\text { mercado de } \\
\text { agua }\end{array}$ & $\begin{array}{c}\% \text { de } \\
\text { crecimiento } \\
\text { de demanda }\end{array}$ & $\begin{array}{c}\text { Demanda } \\
\text { Demanda } \\
2019) * 100 \%\end{array}$ & $0.8 \%$ & $7.9 \%$ \\
\hline
\end{tabular}

Se tiene actualmente un incumplimiento de demanda de aproximadamente 200 bidones retornables B20, lo cual representa un 3\% de la producción total mensual. Según el estándar a incumplimiento de demanda por desabastecimiento de stock, la producción tiene que aumentar en un $7.9 \%$ anual, debido a que, este es el porcentaje de crecimiento que tendrá nuestro sector de agua embotellada en nuestro país [5].

\section{B. Plan de Mantenimiento Preventivo [9].}

\section{1) Objetivo del Plan}

Implementar un mantenimiento preventivo con el fin de mantener la parte mecánica de la máquina Filtro Multimedia para su operatividad cuando la parte electrónica de la máquina esté en óptimas condiciones.

2) Descripción de Actividades de Mantenimiento Preventivo.

- Limpiar del conjunto del conjunto de recogida de agua limpia. Antes de poner en marcha el proceso de purificación y envasado de agua, se procedió a limpiar mensualmente el filtro de recogida de agua. Se implementa este paso, puesto que se procura preservar la línea de vida del filtrante. Se usó como insumos, palos largos para introducirlos dentro del tubo recostado en el piso, conjunto con waype y trapos industriales.

- Inspeccionar las conexiones de la línea hidráulica. Antes de poner en marcha el proceso de purificación y envasado de agua, se procedió a limpiar mensualmente el filtro de recogida de agua. Se implementa este paso, puesto que se procura preservar la línea de vida del filtrante. Se usó como insumos, palos largos para introducirlos dentro del tubo recostado en el piso, conjunto con waype y trapos industriales. 
- Inspeccionar las conexiones eléctricas y los sellos de la caja de control. Consiste en ejecutar la apertura de tapas y verificación de membranas siempre y cuando lo considere oportuno por el técnico de acuerdo con el usuario de la planta. Asimismo, la actividad estuvo a cargo de un especialista en conexiones eléctricas.

- Lubricar la válvula de retro lavado con grasa insoluble. Siendo la función de retro lavado el aspecto más importante de su operación de sistemas de filtro. Sin el retro lavado apropiado, el sucio atrapado no se expulsa. Se considera lubricar la válvula de retro lavado con grasa insoluble al agua. Asimismo, la actividad estuvo a cargo de un especialista en lubricantes, y realizar esta función, se toma tiempo de 2 horas.

- Comprobar si hay fugas en las juntas de acoplamiento. No hace falta algún tipo de instrumento, más que la observación. Asimismo, se ejecutó por un operario de turno.

- Comprobar el diferencial de presión del sistema. En esta actividad se procedió a la configuración del Presión Diferencial en 8 PSI sobre el diferencial de presión del filtro limpio. Asimismo, se ejecutó por un operario de turno.

- Comprobar el caudal del retro lavado. Al efectuar los retro lavados correspondientes, él o los filtros restantes deben continuar produciendo dicho caudal, para mantener la capacidad de la planta. En esta actividad no hace falta algún tipo de instrumento, más que la observación.

- Supervisar la duración del ciclo de descarga. Establecer el tiempo de frecuencia de descarga puede requerir varios días de monitoreo para determinar el ajuste adecuado. En esta actividad no hace falta algún tipo de instrumento, más que la observación.

- Evaluar fluctuaciones estacionales de la calidad del agua. En esta actividad se revisó las fluctuaciones con las que pasa el agua por los diferentes filtros. Basta con observar y reportar anomalías en la secuencia del proceso.

- Limpieza general de la máquina. Conjunto de operaciones que permiten eliminar la suciedad visible o microscópica, enfocándonos en la parte externa de la maquinaria. Estas operaciones se realizaron mediante productos detergentes y materiales elegidos en función del tipo de suciedad y las superficies donde se asienta. Asimismo, la actividad fue ejecutada por el operario de turno.
3) Cronograma de Actividades

TABLA 16

CRONOGRAMA DE ACTIVIDADES

\begin{tabular}{|c|c|c|}
\hline Actividades & $\begin{array}{c}\text { Costo } \\
\text { Anual }\end{array}$ & Frecuencia \\
\hline $\begin{array}{c}\text { Limpiar el filtro del conjunto de } \\
\text { recogida de agua limpia }\end{array}$ & S/ 160 & Mensual \\
\hline $\begin{array}{c}\text { Inspeccionar las conexiones de la } \\
\text { línea hidráulica }\end{array}$ & - & Diario \\
\hline $\begin{array}{c}\text { Inspeccionar las conexiones } \\
\text { eléctricas y los sellos de la caja de } \\
\text { control }\end{array}$ & S/ 80 & Trimestral \\
\hline $\begin{array}{c}\text { Lubricar válvula de retro lavado } \\
\text { con grasa insoluble }\end{array}$ & S/ 230 & Trimestral \\
\hline $\begin{array}{c}\text { Comprobar si hay fugas en las } \\
\text { juntas de acoplamiento }\end{array}$ & - & Semanal \\
\hline $\begin{array}{c}\text { Comprobar el diferencial de } \\
\text { presión del sistema }\end{array}$ & - & Diario \\
\hline $\begin{array}{c}\text { Comprobar el caudal del retro } \\
\text { lavado }\end{array}$ & - & Trimestral \\
\hline $\begin{array}{c}\text { Supervisar la duración del ciclo de } \\
\text { descarga }\end{array}$ & - & Quincenal \\
\hline $\begin{array}{c}\text { Evaluar las fluctuaciones } \\
\text { estacionales de la calidad del agua }\end{array}$ & - & Quincenal \\
\hline Limpieza general de la máquina & S/ 120 & Mensual \\
\hline
\end{tabular}

Para la herramienta de Plan de Mantenimiento Preventivo se utilizó el estándar "Se debe aplicar el método de medición de performance productiva OEE", el cual se basó en la fórmula de OEE calculable con los parámetros de Disponibilidad, Calidad y Rendimiento de la página 23 ubicado en el libro Overall Equipment Effectiveness" [6].

En primer lugar, se indica que se debe determinar la disponibilidad considerando los tiempos productivos e improductivos (tiempo programado y no programado), la calidad mediante la data de producción que cumple con los estándares de calidad (no defectuosos) y la producción total y el rendimiento obtenido del aprovechamiento de la capacidad de la máquina durante su tiempo operativo. Por último, se realiza el cálculo del OEE con los datos resultantes de los parámetros para determinar la efectividad del equipo.

TABLA 17

\begin{tabular}{|c|c|c|c|c|c|}
\hline \multicolumn{6}{|c|}{ ESTÁNDAR DE MANTENIMIENTO PREVENTIVO } \\
\hline Herramienta & Estándar & Indicador & Fórmula & V.A & V.E \\
\hline \multirow{3}{*}{$\begin{array}{l}\text { Mantenimien } \\
\text { to Preventivo }\end{array}$} & \multirow{3}{*}{$\begin{array}{c}\text { OEE: } \\
\text { Overall } \\
\text { Equipme } \\
\text { nt } \\
\text { Effective } \\
\text { ness - } 1^{\text {a }} \\
\text { Ed. } \\
(2006)\end{array}$} & $\begin{array}{c}\text { Horas de } \\
\text { paradas al } \\
\text { mes }\end{array}$ & $\begin{array}{c}\text { Sumatoria de } \\
\text { Horas }\end{array}$ & 2 & 0.65 \\
\hline & & $\begin{array}{c}\text { OEE } \\
\text { mensual }\end{array}$ & $\begin{array}{c}\text { Disp. * Rend. } \\
\text { * Calidad }\end{array}$ & $78 \%$ & $95 \%$ \\
\hline & & $\begin{array}{c}\text { Disponibili } \\
\text { dad } \\
\text { mensual }\end{array}$ & $\begin{array}{c}\text { (H. al mes - } \\
\text { H. Paradas) / } \\
\text { Horas Totales } \\
\text { al mes }\end{array}$ & $95 \%$ & $98 \%$ \\
\hline
\end{tabular}


Los indicadores del problema de paradas de maquinaria son el número de horas totales de paradas al mes, el OEE y disponibilidad mensual. Según el estándar establecido, para el primer indicador el tiempo aceptable es de 0.65 horas totales al mes, mientras que en la actualidad existe un sobretiempo de 2 horas. Posteriormente, para el OEE mensual, se indica que la eficiencia de un equipo para que esté entre los valores del World Class y cuente con una excelente competitividad, debe ser de $95 \%$, sin embargo, se tiene un valor actual de $78 \%$.

Finalmente, en cuanto a la disponibilidad mensual, el cumplimiento debe ser de un $98 \%$, pero se sabe que en la actualidad solo existe un $95 \%$ por lo cual se debe tomar las pautas correctas para el aumento de porcentaje en este indicador.

\section{Kárdex}

Luego de realizar 3 pruebas de error, se determinó que el kárdex fuese llenado por cada trabajador que tenga la función de repartir dicho SKU [10].

TABLA 18

KÁRDEX CONTROL DE ENTRADAS Y SALIDAS (PARTE IZQUIERDA)

\begin{tabular}{|c|c|c|c|c|c|}
\hline Fecha & $\begin{array}{c}\text { Propios } \\
\text { Eberia }\end{array}$ & Terceros & Oficina & $\begin{array}{c}\text { Llenos (en } \\
\text { vehículo) }\end{array}$ & $\begin{array}{c}\text { Salidas } \\
\text { Total }\end{array}$ \\
\hline 14-Oct & 13 & 12 & 11 & & 36 \\
\hline 15-Oct & 15 & & 15 & & 30 \\
\hline 15 -Oct & 0 & & & & 0 \\
\hline $15-$-Oct & 8 & & 7 & & 15 \\
\hline
\end{tabular}

TABLA 19

KÁRDEX CONTROL DE ENTRADAS Y SALIDAS (PARTE DERECHA)

\begin{tabular}{|c|c|c|c|c|c|c|}
\hline $\begin{array}{c}\text { Propios } \\
\text { Eberia }\end{array}$ & $\begin{array}{c}\text { Propios } \\
\text { otras } \\
\text { marcas }\end{array}$ & Terceros & Oficina & Cambios & $\begin{array}{c}\text { Ingresos } \\
\text { Total }\end{array}$ & Obs \\
\hline & & & & & & \\
\hline 12 & & 9 & 7 & 1 & 29 & D1 \\
\hline 14 & & & & & 14 & D2 \\
\hline 14 & & & 11 & & 25 & D3 \\
\hline
\end{tabular}

La función del Kárdex es detallar y apoyar la información que ya recolecta el Control de despacho que la empresa maneja, especialmente en el detalle de las entregas y recojo de los trabajadores de la empresa. La primera columna se especifica la fecha y hora (opcional), en que se realiza un pedido, después de haber sido asignado.

Luego, en el desglose de Salidas Totales, va la cantidad total de bidones que el trabajador saca de la planta en el vehículo. Esta sección se compone de Propios Eberia, que hace referencia a la cantidad total de bidones marca Eberia que dejó en el cliente, la sección terceros hace referencia a la cantidad de bidones que le dio a otro repartidor o distribuidor.

La columna de oficina hace referencia a la cantidad de bidones que dejó en la oficina (ubicada en zona central de Trujillo), y por último la sección llenos, hace referencia a la cantidad de bidones llenos que no ha repartido y siguen estando dentro de su vehículo, luego de haber regresado a la planta de producción. Cabe resaltar que estas últimas columnas mencionadas, fueron llenadas cuando el trabajador haya retornado a la planta de producción.

Por otro lado, tenemos el desglose de las Ingresos Totales, donde irá la cantidad de bidones vacíos que el trabajador deja en la recepción de bidones en la Planta de Producción, luego de haber entregado sus pedidos. Esta sección se compone de Propios Eberia, que hace referencia a la cantidad total de bidones marca Eberia que recogió de clientes, la columna otras marcas, hace referencia a bidones que recogió de clientes, pero no son de marca Eberia (es necesario mapear los bidones de otra marca porque en algunas ocasiones la etiqueta de la marca Eberia no puede cubrir la otra marca; cuando sucede esto, se considera un bidón perdido).

La columna terceros hace referencia a la cantidad de bidones vacíos que está trayendo a la planta de parte de otro trabajador y/o distribuidor. La columna oficina, hace referencia a la cantidad de bidones vacíos que recogió de la oficina (los cuales fueron dejados por algún trabajador, distribuidor y/o ventas). La última sección es Cambios y hace referencia a los bidones llenos y/o semillenos que recogió de un cliente que solicitó un cambio.

La última columna de observaciones solo fue llenada cuando la sección Propios Eberia de Salidas y la suma de la sección Propios Eberia y Propios otra marca de Ingresos, no sea la misma cantidad. Esto indica que le dejó una cantidad de bidones llenos mayor a la cantidad de bidones vacíos que recogió, si la diferencia es negativa. Por el contrario, si la diferencia es positiva hace referencia a que la cantidad de bidones vacíos que recogió es mayor a la cantidad de bidones llenos que dejó en su viaje.

Para el problema de Extravío de Bidones, se decidió utilizar el siguiente estándar, según el artículo 64 de Disminución del inventario final por faltantes de mercancía del decreto nacional 624, el estándar para el faltante de bidones como parte de inventario indica que se puede disminuir hasta en un $5 \%$ de la suma del inventario inicial más las compras, es decir que aún en un $5 \%$ de pérdidas se considera aceptable para el faltante de mercancías de fácil pérdida, como los bidones [7]. 
TABLA 20

ESTÁNDAR DE EXTRAVÍO DE BIDONES

\begin{tabular}{|c|c|c|c|c|c|}
\hline Problema & Estándar & Indicador & Fórmula & V.A & V.E \\
\hline Extravío de & $\begin{array}{c}\text { Decreto } \\
\text { Bidones }\end{array}$ & $\begin{array}{c}\% \text { de } \\
\text { bidones } \\
\text { B20 }\end{array}$ & $\begin{array}{c}\mathrm{N}^{\circ} \text { Bidones } \\
\text { Extraviados / } \\
\mathrm{N}^{\circ} \text { Bidones } \\
\text { extraviados } \\
\text { anuales }\end{array}$ & $6 \%$ & $5 \%$ \\
1989 & $100 \%$ & $5 \%$ & \\
\hline
\end{tabular}

Para el estándar correspondiente el valor estándar máximo permitido del 5\% del decreto 624 de 1989; es decir la empresa debería de perder o extraviar como máximo dicho porcentaje del inventario inicial más otras compras de insumos. Sin embargo, la empresa actualmente tiene un porcentaje de pérdida del $16 \%$ con respecto a la totalidad de bidones que ingresan en un año, siendo perjudicial para las utilidades de la empresa.

\section{RESULTADOS Y DISCUSIÓN}

\section{1) Evaluación Económica}

Para el desarrollo de la evaluación económica se detallan las inversiones realizadas para la implementación de las herramientas (propuestas de solución) de cada problema.

TABLA 21

EGRESOS

\begin{tabular}{|c|c|c|c|c|c|c|}
\hline MES & Dic & Ene & Feb & Mar & Abr & May \\
\hline $\begin{array}{c}\text { Mtto } \\
\text { Preventivo }\end{array}$ & S/ 590 & & & & & \\
\hline Kárdex & S/ 132 & S/ 126 & & & & \\
\hline MRP & S/ 750 & & & & & \\
\hline TOTAL & S/ 1,472 & S/ 126 & S/ 0 & S/ 0 & S/ 0 & S $/ 0$ \\
\hline
\end{tabular}

\begin{tabular}{|c|c|c|c|c|c|c|c|}
\hline Jun & Jul & Ago & Set & Oct & Nov & Dic & TOTAL \\
\hline & & & & & & & S/ 590 \\
\hline & S/ 126 & & & & & & S/ 384 \\
\hline & & & & & & & S/ 750 \\
\hline S/ 0 & S/ 126 & S/ 0 & S/ 0 & S/ 0 & S/ 0 & S/ 0 & S/ 1,724 \\
\hline
\end{tabular}

TABLA 22

BENEFICIOS

\begin{tabular}{|c|c|c|c|c|c|c|}
\hline MES & Dic & Ene & Feb & Mar & Abr & May \\
\hline $\begin{array}{c}\text { Mantenimiento } \\
\text { Preventivo }\end{array}$ & S/ 26 & S/ 26 & S/ 26 & S/ 26 & S/ 26 & S/ 26 \\
\hline Kárdex & S/ 250 & S/ 250 & S/ 250 & S/ 250 & S/ 250 & S/ 250 \\
\hline MRP & S/ 203 & S/ 203 & S/ 203 & S/ 203 & S/ 203 & S/ 203 \\
\hline TOTAL & S/ 478 & S/ 478 & S/ 478 & S/ 478 & S/ 478 & S/ 478 \\
\hline
\end{tabular}

\begin{tabular}{|c|c|c|c|c|c|c|c|}
\hline Jun & Jul & Ago & Set & Oct & Nov & Dic & TOTAL \\
\hline S/ 26 & S/ 26 & S/ 26 & S/ 26 & S/ 26 & S/ 26 & S/ 26 & S/ 308 \\
\hline S/ 250 & S/ 250 & S/ 250 & S/ 250 & S/ 250 & S/ 250 & S/ 250 & S/ 2,998 \\
\hline S/ 203 & S/ 203 & S/ 203 & S/ 203 & S/ 203 & S/ 203 & S/ 203 & S/ 2,432 \\
\hline S/ 478 & S/ 478 & S/ 478 & S/ 478 & S/ 478 & S/ 478 & S/ 478 & S/ 5,738 \\
\hline
\end{tabular}

TABLA 23

FLUJO ANUAL DE CAJA

\begin{tabular}{|c|c|c|c|c|c|c|c|}
\hline \multicolumn{2}{|c|}{ MES } & Dic & Ene & Feb & Mar & Abr & May \\
\hline \multicolumn{2}{|c|}{$\begin{array}{l}\text { FLUJO ANUAL } \\
\text { DE CAJA }\end{array}$} & $\begin{array}{r}-\mathrm{S} / \\
1,472\end{array}$ & $\mathrm{~S} / 352$ & S/ 478 & S/ 478 & $\mathrm{~S} / 478$ & S/ 478 \\
\hline Jun & Jul & Ago & Set & Oct & Nov & Dic & TOTAL \\
\hline $\begin{array}{c}\text { S/ } \\
478\end{array}$ & $\begin{array}{c}\mathrm{S} / \\
352\end{array}$ & $\begin{array}{c}\text { S/ } \\
478\end{array}$ & $\begin{array}{c}\mathrm{S} / \\
478\end{array}$ & $\begin{array}{c}\text { S/ } \\
478\end{array}$ & $\begin{array}{c}\text { S/ } \\
478\end{array}$ & $\begin{array}{c}\text { S/ } \\
478\end{array}$ & S/ 4,014 \\
\hline
\end{tabular}

Mediante la aplicación de las propuestas de mejora se determina que es viable, obteniendo un Tasa Mínima Atractiva de Retorno (TMAR) de $1.53 \%$ obtenido con una tasa de 18\% del Banco Bilbao Vizcaya Argentaria Perú. Se obtiene un TIR de 29\%, el resultado es aceptable porque es mayor a 0 . El VAN de la propuesta es de $\mathrm{S} / 3,496$ y se obtiene un $\mathrm{B} / \mathrm{C}$ de 3.05 .

\section{2) Discusión de Resultados}

Para el primer problema de demanda no satisfecha, según el último "Informe de Mercado de Agua Embotellada", la producción de agua embotellado va a crecer $7.9 \%$ este año [5]. Nuestro valor estándar es de $7.9 \%$ de requisitos cumplidos. Este valor fue comparado con la realidad actual de la empresa, donde ayudándonos con la data de la empresa el mes de noviembre, se tuvo 0.78\%. Luego de implementar el MRP, se llegó a concluir que la producción aumentará un $2 \%$ anual respecto al otro año.

Fig. 2. Comparación de Resultados de MRP

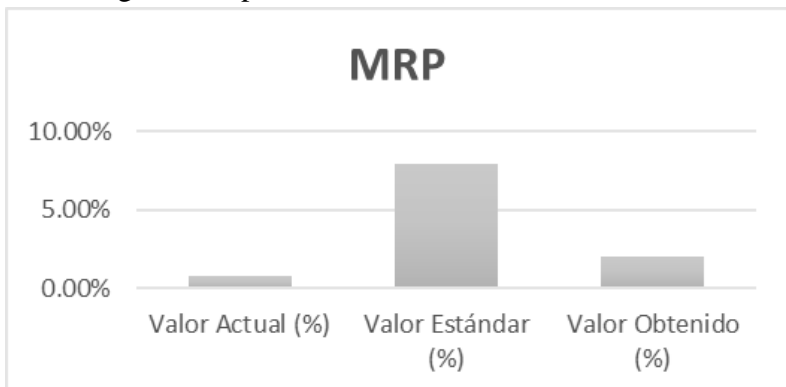

Para el segundo problema de paradas en maquinaria, según el estándar utilizado "Se debe aplicar el método de medición de performance productiva OEE", el cual se basó en la fórmula de OEE calculable con los parámetros de Disponibilidad, Calidad y Rendimiento en el libro Overall Equipment Effectiveness, obtuvimos un valor estándar de 0.65 horas, equivalente a 39 minutos de paradas en maquinaria para obtener un OEE de 95\% [6].

Este valor fue comparado con la realidad actual de la empresa, donde ayudándonos con la data de la empresa el mes de octubre, se tuvo 2.3 horas de paradas de la maquinaria, lo que nos da un valor OEE de $78 \%$. Luego de la implementación se obtuvo un valor de 45 minutos de paradas, que da un valor OEE de $94 \%$. 


\section{Mantenimiento Preventivo}

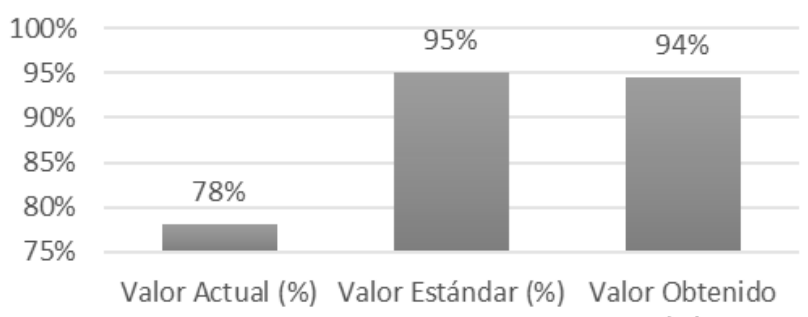

(\%)

Fig. 3. Comparación de Resultados de Mantenimiento Preventivo

Para el tercer problema de bidones B20 extraviados, según el artículo 64 Disminución del inventario final por faltantes de mercancía del decreto nacional 624, el estándar para el faltante de bidones como parte de inventario indica que se puede disminuir hasta en un $5 \%$ de la suma del inventario inicial más las compras, este dato es considerado nuestro valor estándar.

Este valor fue comparado con la realidad actual de la empresa, donde ayudándonos con la data de la empresa el mes de octubre, se tuvo 15 bidones mensuales extraviados, es decir 33\%. Luego de la implementación, obtuvimos un valor de 4 bidones mensuales extraviados, lo que es equivalente a un $9 \%$, teniendo un total de 350 bidones de inventario físico final [7].

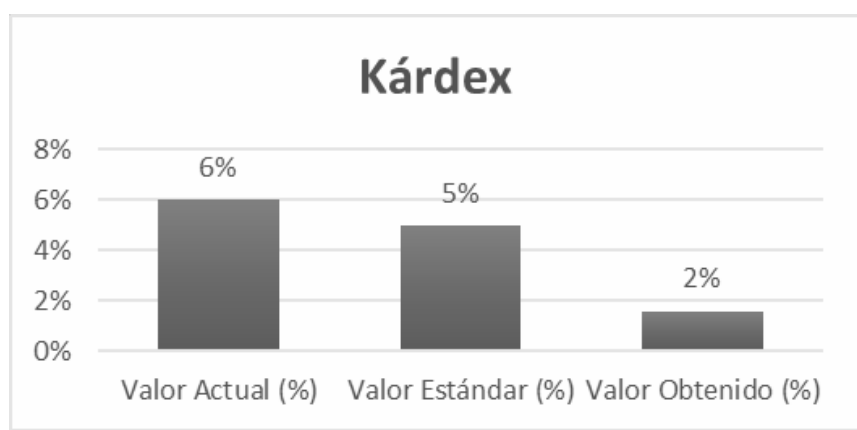

Fig. 4. Comparación de Resultados de Kárdex

\section{3) Conclusiones:}

- Se diseñó e implementó un Plan de Mantenimiento Preventivo y Kárdex en la empresa Corporación Eberia S.A.C, lográndose una reducción anual de S/ 5,231.41.

- Se determinó una alternativa de solución, que consistió en la implementación de las herramientas Plan de Mantenimiento Preventivo, Kárdex y Plan de Requerimiento de Materiales; además se evaluó restricciones como costo de implementación, tiempo de implementación, sostenibilidad, ergonomía, confiabilidad, accesibilidad, aceptación, efectividad, leyes y eficiencia.
- Se diseñaron las herramientas seleccionadas: Plan de Mantenimiento Preventivo, Kárdex y Plan de Requerimiento de Materiales.

- Se identificó estándares apropiados de Ingeniería como el DECRETO SUPREMO No 007-98-SA (100\%), OEE: Overall Equipment Effectiveness - 1a Ed. (2006) (95\%), DECRETO 624 DE 1989 (5\%) e Informe de Mercado de Agua Embotellada (7.9\%).

- Se implementaron las herramientas de solución diseñadas: Plan de Mantenimiento Preventivo, Kárdex y Plan de Requerimiento de Materiales.

- Se evaluó el impacto de la implementación de las herramientas elaboradas, obteniendo un TIR de 18\%, un VAN de S/ 2,198 y un B/C de 1.71.

\section{REFERENCIAS}

[1] R. Jacobs, and R. Chase, Administración de Operaciones. Producción y cadena de suministros, $13^{\mathrm{a}}$ ed., México, D. F.: Editorial Mexicana, 2014, pp. 596.

[2] S. García, Organización y Gestión Integral de Mantenimiento, $1^{\mathrm{a}}$ ed., España, Madrid: Editorial Ediciones Díaz de Santos, S.A., 2003, pp. 32.

[3] C. Mendoza, and O. Ortiz, Contabilidad Financiera para Contaduría y Administración, 23 ${ }^{\mathrm{a}}$ ed., Colombia, Bogotá: Editorial Universidad del Norte, 2016, pp. 238.

[4] P. Belohlavek, OEE. Overall Equipment Effectiveness. Su abordaje Unicista., $1^{\mathrm{a}}$ ed., Argentina, Buenos Aires: Editorial Blue Eagle Group, 2006, pp. 23.

[5] Decreto 624, Artículo 64, Colombia, 30 de marzo de 1989.

[6] M. Díaz, Estadística inferencial aplicada, 23ª ed., Colombia, Bogotá: Editorial Universidad del Norte, 2019, pp. 236.

[7] Informe de Mercado de Agua Embotellada, Maximixe. https://larepublica.pe/economia/1426263-produccion-agua-embotelladacreceria-79-ano/

[8] Bustos Flores, Carlos Enrique, \& Chacón Parra, Galia Beatriz (2007). El MRP En la gestión de inventarios. Visión Gerencial, (1),5-17.

[9] Tavares, Lourival Augusto (2000). Administración Moderna de Mantenimiento. 1era edición, pp. 37.

[10] Carreño Solis, Adolfo Joseph (2017). Cadena de suministro y logística. Fondo Editorial, pp. 26.

[11] Cáceres Carbajal, Claudio Martín (2018). Propuesta de mejora de la eficiencia global de los equipos orientado en el TPM para una empresa envasadora de bebida gasificada no alcohólica. Universidad Peruana De Ciencias Aplicadas.

[12] Llontop Jesús, José; Viacava Campos, Gino Evangelista; Málaga Lasanta, María Isela (2021). Propuesta de mejora del proceso de producción en una planta embotelladora de productos de consumo masivo mediante técnicas Lean. Universidad Peruana De Ciencias Aplicadas.

[13] Moraida Huaca, Ana Gabriel (2016). Implementación de procesos de control de inventarios y su impacto en la rentabilidad de la empresa metal mecánica SERMETAL S.A.C., del distrito de Pataz-La Libertad, 2016. Universidad Privada del Norte. 